\title{
Reflexiones entorno a las aportaciones de José María Izquierdo Arroyo a la representación y la organización del conocimiento: ambición teórica, perspectiva humanística y compromiso académico
}

Reflections around the contribution of José María Izquierdo Arroyo to knowledge representation and organization: theoretical ambition, humanistic perspective and academic engagement

\section{Francisco Javier GARCíA MARCo}

Universidad de Zaragoza

jgarcia@unizar.es

\begin{abstract}
Resumen
Reflexiones sobre la aportación de José María lzquierdo Arroyo al campo de la representación y la organización del conocimiento, realizadas con motivo del homenaje por su jubilación. Se trata de uno de los investigadores españoles en organización del conocimiento que han proporcionado una perspectiva más original e independiente, a la par que sólidamente interdisciplinar, de nuestra disciplina desde fundamentos filosóficos, lógicos, lingüísticos y semióticos. Se destaca especialmente su esfuerzo por contribuir a una síntesis e integración teórica del área de conocimiento desde unas sólidas bases interdisciplinares, así como su perspectiva humanística dialogante, abierta y esperanzadora.
\end{abstract}

Palabras clave: Teoría. Semiótica documental. Lingüística documental. Organización del conocimiento. Representación del conocimiento. Humanidades. José María Izquierdo Arroyo.

\begin{abstract}
Reflections on the contribution of professor José Maria Izquierdo Arroyo to the field of knowledge representation and organization, produced for the festschrift offered to him on the occasion of his retirement. He is one of the most original and independent Spanish thinkers in knowledge organization, who has supported the development of the discipline from his solid foundations in the fields of Philosophy, Linguistics, Logic and Semiotics. His efforts to develop a rigorous, interdisciplinary and integrated theory must be especially highlighted, together with his open and hopeful humanistic perspective.
\end{abstract}

Keywords: Theory. Information Science Semiotics. Information Science Linguistics. Knowledge Organization. Knowledge Representation. Epistemology. Humanities. José María Izquierdo Arroyo.

No se enciende una lámpara para meterla debajo de un cajón, sino que se la pone sobre el candelero para que ilumine a todos los que están en la casa.

(Mt 5, 15)

Es difícil revisar en unas pocas páginas toda una vida dedicada a la investigación académica y a la docencia, pero no lo es tanto el resaltar algunos trazos que me resultan relevantes desde una perspectiva más personal. Para mi, José María Izquierdo representa la aportación exigente, sistemática y con ambición teórica desde las humanidades a la construcción de una ciencia de la información en general y de la organización y representación del conocimiento en particular. A vuelo de águila, su obra supone un gran esfuerzo de integración entre su formación filosófica, literaria y lingüística -a la vez de profundas raíces hispánicas y abierta a las corrien- 
tes más modernas de la ciencia universal- y el proyecto otletiano de construir una ciencia de la documentación.

En estas breves páginas introductorias me centraré en dos aspectos que, a mi modesto entender, tienen una especial importancia para el enfoque de esta revista, que es, estrictamente, la organización y representación del conocimiento, aunque lo transcienden. Ninguno de ellos está de moda en estos tiempos - si se quiere, no forman parte de los frentes de investigación actuales-, pero, precisamente por ello, resulta del mayor interés considerarlos aquí; pues, si nuestra disciplina ha de sobrevivir de forma autónoma, constituyen cuestiones y tareas mayores que habrá que retomar de nuevo, más tarde o más temprano.

Por un lado, José María Izquierdo ha destacado por su gran esfuerzo de sistematización y teorización, buscando construir un sistema disciplinar completo, al que ha denominado semiótica documental. Y lo ha hecho en diálogo con las distintas disciplinas necesarias para establecerlo como una ciencia, esto es, integrándose sólidamente en el sistema general de los conocimientos científicos - con esa perspectiva transdisciplinar que comparte con otra de las grandes figuras de la documentación iberoamericana, Emilia Currás (2008). No en vano, sus obras más señeras son su Concepción lógicolingüística de la documentación, publicada en 1983 - que es un estudio de fundamentación-, sus Esquemas de Lingüística Documental, publicados en 1990 -que constituyen un inventario sistemático de sus recursos teóricos y metodológicos, y de su proyección docente e investigadora-, y La organización documental del conocimiento: marco documental/ corpus otletiano de 1995 - un esfuerzo de profundizar en su pensamiento mediante el diálogo con el teórico que ha constituido su gran referencia intelectual desde el lado de la documentación, Paul Otlet (1934).

Por otro lado, José María Izquierdo destaca como un humanista y un académico en un entorno y en una disciplina -la biblioteconomía y la documentación- en la que tantas veces se ignoran las fuentes y el estado de la cuestión, abandonándose así las formas que han soportado tradicionalmente la actividad académica como un esfuerzo sostenible y duradero en el tiempo. En estos años de crisis de nuestra economía y de nuestra vida política $-\mathrm{y}$, dentro de ellas, como no podía ser de otra manera, de nuestro sistema universitario y de nuestro área de conocimiento (García Marco, 2013) - su obra constituye una llamada a profundizar, a buscar raíces y a edificar sobre sólidos cimien- tos, pues sólo así se puede constituir una disciplina que se proyecte hacia el futuro, en vez de prodigarse en esfuerzos dispersos o fragmentados, condenados a desaparecer en los torbellinos e inundaciones de la investigación científica.

\section{La ambición de la teoría}

Conocí al profesor José María Izquierdo personalmente hace ya algo más de veinte años durante el I Encuentro de Organización del Conocimiento en Sistemas de Información y Documentación, que celebramos en Madrid en la histórica Residencia de Estudiantes el 4 y 5 de noviembre de 1993. Habíamos preparado cuidadosamente el encuentro para que sirviera de lanzamiento del Capítulo Español de la Sociedad Internacional para la Organización del Conocimiento, cuya fundación formal tuvo precisamente lugar en dicha reunión. Era un ambiente agradable y directo, más propio de los seminarios científicos que de los congresos.

Ya habiéndonos presentado la noche anterior, coincidimos en la primera sesión, el día 4 a las 10:30, dedicada a los Fundamentos teóricos y la investigación básica. Yo presenté una ponencia sobre la Complementariedad de los paradigmas cognitivo y semiótico-lingüístico en la construcción de un modelo de representación y recuperación del conocimiento, que luego se publicó en las actas como Paradigmas científicos en recuperación de la información (García Marco, 1993). En la propuesta, distinguía entre los paradigmas tradicional, fisicalista, semióticolingüístico, lógico-matemático y cognitivo, como paradigmas mayores; y, como emergentes o menores, los economicista, sociologista e historicista. José María Izquierdo intervino nada más acabar para decirme que el paradigma semiótico-lingüístico, el lógico-matemático y el cognitivo podían formar un solo paradigma, y que incluso alguno más podía integrarse en él.

Se trataba de algo más que una anécdota. Efectivamente, toda su vida académica dentro de nuestra área ha sido un gran esfuerzo para acercar esas perspectivas dentro de una teoría común, una ambiciosa tarea para la que estaba tan bien preparado por su sólida trayectoria anterior como profesor de lógica, filosofía y crítica literaria, que le proporcionaba una manera diferente de abordar los problemas de la organización y representación del conocimiento.

Aunque fue ese año cuando conocí personalmente a José María, por supuesto, había leído con cuidado algunas de sus obras publicadas, en particular, su Concepción Lógico-Lingüística de la Documentación (Sagredo e Izquierdo, 
1983b) y las publicaciones relacionadas (Sagredo e Izquierdo, 1982a, 1982b, 1989ab).

No había leído, sin embargo, todavía sus Esquemas de Lingüística Documental (Izquierdo, 1990abc) en los que desplegaba el trabajo de tantos años realizado en pos de una disciplina integrada del tratamiento documental de contenido, desde la lingüística documental y con el horizonte puesto en la semiótica documental. Se trataba de un trabajo ingente, que junto con los de Antonio Luis García Gutiérrez (v. g. 1984, 1990, 1998), José Antonio Moreiro González (v. g. 1993, 2004) y algunos otros autores especialmente, en Brasil, del grupo Temma y de la escuela de Marília, creada por José Augusto Chaves Guimarães - ha sentado las bases en nuestro entorno para la construcción de una semiótica documental, un proyecto que, cuando se estabilice Internet habrá que retomar de forma prioritaria como programa general de investigación, aunque posiblemente con nuevas alianzas.

Sin duda alguna, el estudio de las bases lingüísticas y semióticas de la organización del conocimiento es una de su claves, junto con las ontoloógicas y cognitivas. Sirva como ejemplo el hecho de la teoría de facetas - tan paradigmática en nuestra disciplina- no deja de ser una aplicación simplificada de la gramática tradicional a la recuperación temática de la información (1), en la que se construyen frases normalizadas y predecibles que permiten recuperar temas complejos en un universo informacional caracterizado por la rápida y permanente expansión durante los últimos siglos.

Pero además de por su amplia perspectiva interdisciplinar y por su voluntad teórica, José María Izquierdo destaca también por su pensamiento independiente. Otra de las características más importantes de su obra es precisamente que, para defender esa necesidad de integración y dar coherencia sistemática a un campo tan fragmentado como el de la organización y representación del conocimiento, no le ha importado expresar opiniones alejadas de las modas del momento, proyectadas desde la sólida perspectiva humanística que le ha caracterizado siempre. Ejemplo de su aportación crítica ha sido su análisis de los tesauros (Izquierdo y Moreno, 1992, 1994, 1995), que, a su entender, sólo darán todo el fruto prometido en sus ambiciosos objetivos si incorporan mayor complejidad lingüística y lógica.

Otra gran virtud de José María Izquierdo como teórico ha sido adoptar una perspectiva auténticamente centrada en el conocimiento - no en la información, en el sentido del paradigma fisica- lista-. Efectivamente, la organización del conocimiento no consiste sólo en crear índices que apuntan a fragmentos de información, sino en trabajar los mensajes para re-relacionarlos de una forma sistemática y capturar el conocimiento que existe en ellos de una forma organizada y predecible que permita su reutilización, así como, más allá, la acumulación sólida, crítica y civilizada del conocimiento. José María Izquierdo captura esta idea de una forma muy original con su concepto de archidocumento, que emerge de con sus profundos conocimientos de crítica textual y literaria. Nos muestra como los documentos tienen una estructura hacia dentro y una estructura hacia fuera; $y$, por tanto, la organización del conocimiento tiene que explorar, relacionar y ayudar a construir ambas dimensiones de forma sistematica.

Es cierto que el proyecto de una semiótica documental integradora o de una teoría integrada de la representación y organización del conocimiento se enfrenta a dos grandes escollos, que resultan difíciles de remover.

En primer lugar, las disciplinas humanísticas y sociales que interesan de una manera más directa a la organización del conocimiento se encuentran cada vez más fragmentadas, cosa que por otra parte sucede con las humanidades en su conjunto. El último gran esfuerzo de unificarlas basado en la metodología y algunos principios comunes -el estructuralismo- se ha roto en un ecosistema de esfuerzos difícil de articular, y, en muchos casos, refractario a cualquier golpe de timón cuyo objetivo sea la coherencia y la sistematicidad, pues sus horizontes son precisamente los contrarios. Si el objetivo de las humanidades es conseguir un saber integrado, holístico y transdisciplinar, está claro que su misma misión queda en peligro al no alcanzarlo; pues para conseguir resultados fragmentarios de forma eficaz, la aplicación del método científico estricto parece ciertamente superior.

Por otro lado, el discurso teórico y aún metodológico no se considera hoy en día merecedor del menor interés si no sirve al menos para dar cobertura de mercadotécnica a las aplicaciones, notablemente a aquellas que proporcionan rentas o votos. Y el problema es que el propio énfasis en lo práctico y pragmático bloquea muchas veces el avance en lo teórico y sistemático, cuyos beneficios - facilitan la formación y el estudio al eliminar redundancias, incoherencias y resaltar lagunas - requieren de un plazo más largo, alejado de las urgencias del momento. Como señaló Peter Jaenecke (1997) la mejor forma de organización del conocimiento es la creación de teorías sistemáticas. 
Por ello, es necesario 'metaconversar' nuestra discusión en un plano más elevado: la consideración de las dimensiones filosóficas de la organización y representación del conocimiento; si se quiere, de su fundamentación desde una perspectiva humanística.

\section{La perspectiva humanística}

Tradicionalmente, y de forma más insistente en los últimos años, la documentación ha sido revindicada repetidamente como ciencia aplicada. Como resultado se ha desarrollado mayormente desde una perspectiva técnica, a veces tecnicista, centrada en el desarrollo de medios y técnicas al servicio de fines que quedaban cada vez más alejados del objeto de la ciencia de la información como tal. De hecho, en el caso concreto de España, la dimensión humanística que se proporcionaba al inicio de los estudios de Biblioteconomía y Documentación se ha ido perdiendo en el tránsito a la Información y Documentación, hasta casi desaparecer.

El pensamiento y la formación humanística no pueden sustituir la perspectiva científica estricta -la contrastación con los hechos y los procedimientos de investigación inductivos y experimentales - ni la técnica —el desarrollo de métodos y máquinas para resolver tareas prácticas-, pero constituye un pilar necesario para sustentar la investigación, la enseñanza y el aprendizaje en cualquier campo, y, de forma muy especial, en la organización del conocimiento, proporcionando la perspectiva de los fines y de lógica antropológica subyacente.

José María Izquierdo lo expresa de forma contundente en este mismo volumen, refiriéndose a la filosofía, la disciplina cumbre de la síntesis humanística (Izquierdo Alonso e Izquierdo Arroyo, 2014):

La Filosofía propiamente tal y bien entendida, que no la "académica", ha de estar presente en todas las ciencias y especialidades. Aporta una dimensión de profundidad y radicalismo a todas las preguntas e investigaciones. Más allá de los "datos" y de los "hechos", se cuestiona por los porqués y paraqués de cuanto nos rodea.

También el experto en desarrollo de recursos humanos Stephen Covey (1989, p. 332-333) profundiza en la misma dirección, planteando la diferencia radical entre el adiestramiento técnico y la auténtica educación:

Resulta extraordinariamente valioso adiestrar la mente para que tome distancia respecto a su propio programa, y lo examine. Esa es para mí la definición de la educación humanística: la capacidad para examinar los programas de la vida, en el marco de otros paradigmas, y de los interrogantes y propósitos de mayor alcance. El adiestramiento, sin una educación de ese tipo, estrecha y cierra la mente pues los supuestos subyacentes de ese mismo adiestramiento nunca son objeto de examen. Por ello, es tan valioso leer con amplitud y exponerse a los grandes pensadores.

En realidad, adoptar una perspectiva humanística significa reconocer que no es posible tratar la realidad humana de forma reduccionista y fragmentaria, sino, por el contrario, de modo sistemático y holístico, dentro de una sólida tradición, pero sin miedo a la crítica y los avances.

Al fin y al cabo, las humanidades buscan comprender al ser humano -el yo, el tú, el otroen su contexto y sus relaciones, y proponer caminos para vivir mejor basados en ese conocimiento tanto en el plano del comportamiento correcto como en el de la construcción de una vida y un entorno hermoso. Esto es, buscan al menos una física - un entendimiento del medio ambiente del hombre-, una antropología —una comprensión del ser humano-, una ética unas normas de desenvolvimiento personal y comunitario-, y una estética - una aproximación óptima a la creación-, que se corresponden con los valores de la verdad, el bien y la belleza, sintetizados y codificados en el pensamiento occidental desde Platón. Se trata, en suma, de que los cuatro planos del ser humano -medio, conocimiento (2), comportamiento y resultado transformador - se desarrollen de la mejor forma posible. Aunque tan alta ambición choca con la naturaleza limitada de los seres humanos - que es capaz de contemplar lo mejor y aspirar a ello, pero que no está a la altura de conseguirlo totalmente, porque, en distinta medida, caemos en la mentira, la maldad y la dejadez- no por eso dichos objetivos son menos importantes, pro el contrario, son faros que dan sentido al esfuerzo e iluminan el camino.

En nuestra disciplina, además, es especialmente necesario el recurso al pensamiento humanístico e interdisciplinar; pues el campo de la organización del conocimiento no permite una separación tan limpia entre medios y fines como otras ciencias y, en concreto, como otras subdisciplinas de la ciencia de la información. Lo que la hace diferente es que necesita situarse en la mente de los usuarios y profundizar en sus esquemas cognitivos para construir instrumentos de mapeo de los conceptos que usan para aprender la realidad y, secundariamente, de los términos que utilizan para comunicar dichos conceptos. $Y$ dichos conceptos están íntimamente conectados con sus fines. De hecho, en la práctica, son los fines diferentes los que explican la existencia de organizaciones del conocimiento distintas para un mismo dominio. Es la 
existencia de objetivos, perspectivas y pragmáticas diferentes la que determina que se opte por un esquema clasificatorio $u$ otro. A veces es solo una cuestión de orden, de valoración ontológica, que siempre termina siendo al final axiológica.

Pero, además de las ontológicas y epistemológicas, también hay razones contextuales, de lógica histórica, que exigen volver nuestra vista hacia las humanidades. Aunque, hasta un determinado punto, es posible deslindar la física de la filosofía, una vez establecidos sus presupuestos metafísicos y sus bases epistemológicas y ontológicas; no es, sin embargo, viable separar una disciplina humana aplicada de la reflexión permanente sobre los fines, porque, como consecuencia, se verá privada de orientación y energía en cuanto se agote el paradigma dominante.

Y precisamente, se empiezan a vislumbrar en el horizonte los límites del paradigma actual, orientado a los aspectos técnicos del tratamiento documental de información y basado en la incorporación de las nuevas tecnologías de la información y la comunicación y de las técnicas de gestión social y empresarial, que de por sí han constituido grandes avances. En una sociedad en la que los competidores de los trabajadores humanos no son ya tanto otros seres humanos, sino cada vez más los bots y robots, la importancia de los trabajos técnicos y administrativos disminuyen como competencias que permiten conseguir puestos de trabajo $y$, en general, generar actividad humana. En este nuevo entorno, las humanidades -especialmente las aplicadas - vuelven a ser una fuente de trabajo, porque constituyen un aspecto que no es reemplazable por automatismos. La organización del conocimiento en particular se mantiene como una fuente de trabajo netamente humanista y difícilmente automatizable, aunque, por su puesto, se vea inevitablemente beneficiada, apoyada e incluso requerida por las tecnologías de la información y la comunicación.

Además, las disiciplinas humanísticas permiten desarrollar, cuando se cultivan adecuadamente, las competencias "genéricas" que constituyen hoy la mejor garantía de empleo para los estudiantes. En particular, la organización del conocimiento, desde una perspectiva humanística, constituye un espacio intelectual ideal para desarrollar la creatividad, el trabajo en equipo, el rigor, la comprensión de los demás, la capacidad de comunicación, la iniciativa, el manejo de las fuentes de información y la orientación a resolver problemas que tanto se demandan actualmente (Moreiro, 2014; Wagner, 2008). En ese contexto, la vuelta a las humanidades en la ciencia de la información constituye una dimensión necesaria, y resulta relevante e incluso necesario un programa de rehumanización de la organización del conocimiento, y de su enseñanza, sin descuidar sus aspectos científicos y técnicos.

No sería justo dejar sin señalar que la perspectiva humanística también tiene sus sombras, en particular la tendencia al dogmatismo, al idealismo alejado de la realidad empírica y el recurso estético a una erudición alejada de cualquier compromiso. Sin embargo, es posible adoptar una posición humanística protegida de estos defectos; y de hecho, José María Izquierdo ha sido un ejemplo a lo largo de su vida académica de cómo conjurar todos estos peligros con efectividad, desde el pluralismo metodológico, la perspectiva filosófica, el rigor lógico, el permanente contraste de ideas contrapuestas aunque personalmente no las asumiera- y la referencia a los hechos y a las aportaciones anteriores.

\section{La organización del conocimiento como disciplina académica en una universidad en tránsito}

Además de su aportación a la organización del conocimiento, José María Izquierdo constituye, desde una perspectiva académica, una referencia clara y honesta dentro de este gran proceso de cambio que se ha producido durante estos años entre dos modos de entender la academia y la vida universitaria (3); y, por ello, su fecundo trabajo, recogido en tantas publicaciones, será siempre una invitación permanente a la reflexión sobre nuestra tarea como docentes e investigadores.

José María es un egregio representante en nuestro área de conocimiento de una generación de profesores que está dejando las aulas y los despachos universitarios, llevándose con ellos una forma de entender la vida académica, la docencia y la investigación muy diferente a la que se está ahora consolidando entre nosotros.

La universidad de la que venimos es la de los profesores volcados en la vida académica y universitaria, dedicados a formar alumnos con un conocimiento profundo y sistemático de un área del conocimiento, algunos de los cuales habrían de proseguir la labor de acrecentar y depurar el legado recibido y de formar a las nuevas generaciones. La vida académica de entonces pivotaba entorno a grandes momentos en los que brillaba con todo su esplendor: las tesis de grado y doctoral, y las memorias de acceso a la titularidad y la cátedra. Era otro mundo, en el que se intentaba unir sólidamente investigación 
y docencia mediante programas docentes e investigadores orientados a proyectos de larga duración, donde la estructura clave de organización era el seminario de investigación y la cátedra, y bajo gobiernos universitarios ciertamente paternalistas, pero livianos en la práctica por su escaso tamaño y poder efectivo.

El modelo de universidad al que vamos - ¿en el que estamos? - parece ser cada vez más el de la corporación sociedad anónima, fruto probablemente de las presiones de la globalización. La universidad contemporánea — cada vez más volcada hacia la vida política, empresarial y profesional, hasta casi el descentramiento- ha tomado una consistencia líquida. En ella, los docentes e investigadores tienen que navegar los continuos cambios, acreditando continuamente su carrera, sus proyectos y sus publicaciones, en una cadena de actividades y resultados cada vez más fragmentaria y fractal. Respecto al gobierno universitario, se aprecia una incipiente ruptura desde la colegialidad hacia una estructura de niveles muy separados bajo burocracias universitarias cada vez más pesadas, dedicadas a facilitar el control del profesorado más independiente y la explotación del más productivo.

Entre medio hemos disfrutado -y sufrido- de un enorme crecimiento. Creció el número de estudiantes -el gran bien de la universidad democrática-; y aprovechando las velas llenas de ese viento, lo hicieron también las plantillas, las titulaciones y la burocracia universitaria. En medio siglo, el mundo, Europa y España se han ido llenando de más universidades, cada vez más grandes. Pero el sistema parece haber alcanzado los límites del rendimiento decreciente. Ahora que hay menos estudiantes - porque nacen menos y porque los que podría haber están desmotivados por un coste difícilmente asumible o una relación desventajosa entre esfuerzo y recompensa-, está surgiendo un nuevo consenso de que sobran universidades, departamentos y profesores; y los Estados quieren reconvertirlas en instrumentos de investigación, desarrollo, innovación y formación al servicio del tejido productivo. Pero este nuevo horizonte constituye un viraje muy importante respecto de la misión histórica de la universidad, que no parece posible sin que previamente se produzca una gran poda de la actual.

En estos tiempos de tránsito, José María ha sido siempre para mi un gran ejemplo del profesor académico, que se esfuerza sin denuedo por compaginar los retos de la educación universitaria de masas con las necesidades de una investigación cada vez más aplicada especialmente en una disciplina como la nues- tra-, pero de una manera compatible con los valores y los modos de la universidad clásica en la larga duración, que diría Braudel (1958).

Es cierto que cualquier tiempo anterior parece mejor de lo que fue a los que lo recordamos con la suficiente lejanía; también que el futuro que se sueña en la plenitud de la ilusión raramente llega con los nítidos colores con los que se imaginó; pero, sobre todo, es verdad que el presente sólo puede ser auténticamente grande si se construye - la parte que es posible- desde la conciencia de la misión a largo plazo. Por ello, muchas gracias, profesor José María Izquierdo, por habernos animado, acompañado y enseñado en el esfuerzo de construir la organización y la representación del conocimiento como una disciplina humanística, esto es, inmersa dentro de un proyecto mayor, que hunde sus raíces en la fértil tierra del pensamiento occidental y nos proyecta con ilusión y coherencia hacia el futuro, por encima de las turbulencias del presente y de sus prometedoras pero agitadas promesas.

\section{Notas}

(1) Ranganathan utiliza una frase del tipo sujeto/objeto (dependiendo de si es personal o impersonal), predicado (proceso) y complementos circunstanciales de material, tiempo y espacio como categorizador universal de los temas.

(2) Conocimiento hacia dentro y hacia fuera, hacia las personas, los seres y las cosas.

(3) En España están apareciendo continuamente títulos sobre la reforma universitaria, pero asombra leer la ácida, caricaturesca, a veces exaltada, pero premonitoria crítica del tránsito entre el modelo académico de la universidad y el emergente que realizó ya Sykes (1988) para el caso estadounidense. Más de dos décadas después, su lectura hace pensar sobre lo que está pasando en nuestro contexto iberoamericano, que intenta adaptarse a los cambios en la educación superior que se han producido en las sociedades más avanzadas.

\section{Referencias}

Braudel, Fernand (1958). Histoire et sciences sociales: La longue durée. // Annales: Histoire, Sciences Sociales. 13:4 (October-December 1958) 725-753.

Covey, Stephen R. (1994). Los 7 hábitos de la gente eficaz : la revolución ética en la vida cotidiana y en la empresa. Barcelona, etc.: Paidós.

Currás Puente, Emilia (2008). Ciencia de la información bajo postulados sistémicos y sistemáticos. Caracas: Edición personal.

García Gutiérrez, Antonio (1984). Lingüística documental : [aplicación a la documentación de la comunicación social]. [Barcelona]: Mitre.

García Gutiérrez, Antonio Luis (1998). Principios de lenguaje epistemográfico : la representación del conocimiento sobre Patrimonio Histórico Andaluz. [Sevilla]: Instituto Andaluz del Patrimonio Histórico.

García Gutiérrez, Antonio Luis (1990). Estructura lingüística de la documentación : teoría y método. Murcia: Universidad de Murcia, Servicio de Publicaciones. 
García Marco, Francisco Javier (1995). Paradigmas científicos en recuperación de la información. // Organización del Conocimiento en Sistemas de Información y Documentación : 1 : Actas del Primer Encuentro del Capítulo Español de la Sociedad Internacional para la Organización del Conocimiento. Zaragoza, Universidad. 99-112.

García Marco, Francisco Javier. Educación y aprendizaje de la información y la documentación: raíces, desafíos y líneas de acción. En: El profesional de la información. 22: 6 (2013) 489-504.

Jaenecke, Peter (1997). Knowledge organization due theory to theory formation. // Organización del conocimiento en sistemas de información y documentación. 2 39-55.

Izquierdo Arroyo, José María (1990). Esquemas de Lingüística Documental. Barcelona: PPU. 3 vols.

Izquierdo Arroyo, José María (1992). Cuatro trabajos en curso. // Documentación de las Ciencias de la Información. 15 (1992) 35-65.

Izquierdo Arroyo, José María (1995). La organización documental del conocimiento: 1/1: El marco documental. Madrid: Tecnidoc.

Izquierdo Arroyo, José María; Moreno Fernández, Luis Miguel (1992). Diseño de una base de datos de prensa, controlada por un lenguaje facetado de estructura combinatoria 'thesaurus'. //. Revista Española de Documentación Científica. 15:1 (1992) 44-63.

Izquierdo Arroyo, José María; Moreno Fernández, Luis Miguel (1995). Problemas de terminología metalingüística en los lenguajes documentales de estructura combinatoria. // García Marco, F. J. (ed.). Organización del Conocimiento en Sistemas de Información y Documentación, 1. (Actas del I Encuentro de ISKO-España, Madrid 4-5 Nov. 1993). Zaragoza, 1995. 51-63.

Izquierdo Arroyo, José María; Moreno Fernández, Luis Miguel (1994). Listas de encabezamientos de materia y Thesauri en perspectiva comparada. // Documentación de las Ciencias de la Información. 17 (1994) 287-310.

Moreiro González, José Antonio (2004). El contenido de los documentos textuales : su análisis y representación mediante el lenguaje natural / José Antonio Moreiro González. Gijón: Trea.
Moreiro González, José Antonio; Izquierdo Arroyo, José María (1993). Aplicación de las ciencias del texto al resumen documental. Getafe, Madrid: Universidad Carlos III.

Moreiro-González, José-Antonio. Aún en torno a la formación universitaria, el empleo y la recesión. // Anuario ThinkEPI. 8 (2014) 15-23.

Otlet, Paul; La Fontaine, Henry (1934). Traité de documentation: le livre sur le livre: théorie et practique. Bruxelles: Mundaneum.

Sagredo Fernández, Félix; Izquierdo Arroyo, José María (1982a). Reflexiones sobre Documento: palabra/objeto. // Boletín Millares Carlo. 3:5 (1982) 161-197.

Sagredo Fernández, Félix; Izquierdo Arroyo, José María (1982b). Análisis formal de las definiciones de Documentación. // Boletín Millares Carlo. 3/6 (1982) 239-287.

Sagredo Fernández, Félix; Izquierdo Arroyo, José María (1989a). Análisis formal de las definiciones de Documentación. // López Yepes, J. (comp.). Fundamentos de Información y documentación. Madrid: EUDEMA. 160200.

Sagredo Fernández, Félix; Izquierdo Arroyo, José María (1989b). La concepción ordinaria de la Ciencia de la Documentación. // López Yepes, J. (comp.). Fundamentos de Información y documentación. Madrid: EUDEMA. 5377.

Sagredo Fernández, Félix; Izquierdo Arroyo, José María (1983b). Concepción lógico lingüística de la Documentación. Madrid: IBERCOM.

Sagredo Fernández, Félix; Izquierdo Arroyo, José María (1983). Concepción lógico-lingüística de la documentación. Madrid: Universidad Complutense de Madrid, Departamento de Documentación.

Sykes, Charles J. (1988). ProfScam: professors and the demise of higher education. New York: St. Martin's Press.

Wagner, Tony (2008). The global achievement gap: Why even our best schools don't teach the new survival skills our children need and what we can do about it. New York: Basic Books. 
